\title{
SISTEMATIZACIÓN DE UNA EXPERIENCIA PEDAGÓGICA EN EL AULA. EL DIÁLOGO DE SABERES Y LA INTERCULTURALIDAD COMO PILARES EN LA FORMACIÓN DE LÍDERES Y SUJETOS
}

\section{SYSTEMATIZATION OF A PEDAGOGICAL EXPERIENCE IN THE CLASSROOM. THE DIALOGUE OF KNOWLEDGE AND INTERCULTURALITY AS PILLARS IN THE TRAINING OF LEADERS AND SUBJECTS}

\section{Artículo de reflexión}

Mónica María Castaño Castaño, Lady Jhoanna García García, Alberto José Echeverría Reina, Ana María

Becerra Ocampo y Henry Alonso Ocampo Marín

Diplomado en Docencia Universitaria

Institución Universitaria Antonio José Camacho

Recibido: 05/03/2020 Aprobado: 27/04/2021

Cómo citar este artículo:

Castaño Castaño, M.M., García García, L.J., Echeverría Reina, A.J., Becerra Ocampo, A.M. y Ocampo Marín, H.A.(2021). Sistematización de una experiencia pedagógica en el aula. El diálogo de saberes y la interculturalidad como pilares en la formación de líderes y sujetos. Revista Sapientía, 13(25), 78 - 89.

\section{RESUMEN}

En el presente artículo se presenta el reto de pensar el proceso de formación de líderes y sujetos mediante el diálogo de saberes. En este sentido, se reflexiona una experiencia docente, desde la cultura e identidad de los individuos en formación. Se piensa sobre sus conocimientos, sentimientos y acciones y se propone el sentido que debe cobrar la educación desde la sistematización de una experiencia profesoral como un proceso de reflexión e interpretación crítica y autocrítica sobre la práctica en el aula.

\section{PALABRAS CLAVE}

Corrientes pedagógicas, Didácticas contemporáneas, Potenciales del desarrollo humano, Sistematización de experiencia, Metacognición

\section{ABSTRACT}

In this article, the challenge of thinking about the process of training leaders and subjects through the dialogue of knowledge is presented. In this sense, a teaching experience is reflected, from the culture and identity of the individuals in formation. They think about their knowledge, felings and actions and propose the meaning that education should charge from the systematization of a teaching experience as a process of reflection and critical and self-critical interpretation about classroom practice.

\section{KEYWORDS}

Pedagogical Currents, Contemporary Didactics, Potentials of Human Development, Systematization of Experience, Metacognition.

\section{INTRODUCCIÓN}

El presente artículo de sistematización de experiencias es el resultado del proceso de observación y análisis de la experiencia pedagógica llevada a cabo durante el desarrollo del Diplomado de Docencia Universitaria de la cohorte de julio a diciembre del 2018, el cual da cuenta de los alcances en formación y desarrollo en torno a la 
necesidad de reflexionar y analizar el quehacer docente y establecer nuevas formas de actuación en el contexto del aula, desde una mirada crítica y potenciadora de la gestión del conocimiento y del fomento de los potenciales del desarrollo humano.

En dicha experiencia pedagógica se asume el reto de comprender el acto de enseñar y de aprender a través de la observación directa en el aula y, posteriormente, de manera colectiva, con reflexión crítica, integrar los hallazgos bajo el modelo de sistematización de experiencia pedagógica, en donde se relacionan conceptos, se construye un discurso en torno a los hallazgos en didáctica y se sitúa la práctica docente en el ámbito de la revisión permanente, en diálogo con el discurso de la pedagogía, la educación y la enseñanza en bien de la construcción de conocimiento bajo la orientación de las didácticas contemporáneas encaminadas a la transformación del sujeto estudiante y del sujeto profesor.

Se entiende por sistematización en el contexto del aula aquella reflexión crítica de la práctica pedagógica que actúa como instrumento de mejoramiento continuo del profesor y de su discurso pedagógico, donde la acción, la práctica, la experiencia y la praxis intentan establecer un diálogo permanente entre la experiencia didáctica y los procesos de enseñanza y aprendizaje ${ }^{1}$.

Marco Raúl Mejía, uno de los autores colombianos más influyente en temas de sistematización de experiencias, afirma que:

hablar de la sistematización es hablar de una práctica investigativa que se ha venido conformando en América Latina en la segunda mitad del siglo XX y los comienzos de este nuevo milenio. En ese sentido, hablamos de un proceso que nació unido a las dinámicas sociales, políticas $y$ culturales que se desarrollaron en nuestra realidad y que llegaron a otras latitudes, especialmente en Asia y África. (Mejía, 2007)
El modelo de Sistematización de la experiencia pedagógica implementado en el aula y propuesto por los profesores del Diplomado en Docencia Universitaria de la Institución Universitaria Antonio José Camacho, recoge la discusión pedagógica entre las didácticas tradicionales y las contemporáneas, y enfoca la atención en el aprendizaje y en el sujeto que aprende, permitiendo que la gestión del conocimiento se dé desde la apropiación en el aula de los dispositivos básicos del aprendizaje y desde las lógicas del desarrollo de los potenciales: comunicativo, socio-afectivo, ético, político y cognitivo, otorgándole un lugar significativo al profesor en su práctica docente.

La sistematización de la experiencia se llevó a cabo atendiendo el modelo de sistematización antes mencionado, desde tres unidades de análisis: Unidad de Contexto, la cual hace referencia a los aspectos que conforman el andamiaje necesario e indispensable en el desarrollo de una experiencia de aprendizaje en el aula, esta unidad tiene en cuenta la caracterización del grupo, el ambiente del aula, las condiciones y desarrollo de la clase; se incluye además la planeación y el análisis metacognitivo, los procesos implementados para la gestión del conocimiento al igual que la inclusión de las didácticas contemporáneas. Unidad de Sentido, en esta se relacionan dos procesos significativos: la interpretación de la práctica en el aula, donde gana sentido el proceso de observación individual y la correspondiente reflexión de cada uno de los docentes, unido a la teorización de la práctica, es decir, al hallazgo y asociación de conceptos válidos dentro del proceso de sistematización; un elemento fundamental en ambos procesos es la reflexión colectiva que gana sentido en la medida en que orienta la discusión, el diálogo y el intercambio de saberes. Finalmente, la Unidad de Transformación, que indica, dentro de la reflexión colectiva, lo novedoso y significativo de la práctica docente, las cuales guían el proceso de sistematización

\footnotetext{
1 Planteamiento formulado por el profesor Carlos Arturo Muñoz en la clase de sistematización de experiencias pedagógicas del Diplomado en Docencia Universitaria de la Institución Universitaria Antonio José Camacho.
} 
de la práctica docente e indican los pasos a seguir dentro del análisis que conlleva a una mirada retrospectiva y transformadora del lugar del profesor en el aula y del sentido y significado de su papel como agente transformador y generador de conocimiento.

Por tal motivo, en esta experiencia pedagógica se precisa una revisión de la práctica docente donde la observación individual y el análisis colectivo e interdisciplinario dan lugar a una interpretación del acto de aprender, un análisis teórico que conlleva a un diálogo de diferentes posturas y a una construcción en conjunto de una serie de relaciones conceptuales que privilegien el contexto, el cual adquiere sentido y significado en la medida que propicie la generación de procesos transformadores de la práctica docente en el aula.

Desde este punto de vista se reafirma que el profesor universitario se asume como un profesional reflexivo, un intelectual crítico, además de maduro, comprometido y definido, tal vez con militancia política, con una pedagogía contextuada y que tiene clara conciencia que su papel es el de transformador social y guía de las nuevas generaciones ${ }^{2}$.

Este artículo enfoca su atención en establecer las relaciones entre las tres unidades de análisis y permitirá dar cuenta de los procesos de aprendizaje de los sujetos en formación (profesor y estudiantes), en torno a la inclusión de una mirada integral e integradora de los procesos de enseñanza desde las didácticas contemporáneas, la reflexión y teorización sobre la práctica en el aula, con el objeto de priorizar la interculturalidad, el aprendizaje basado en problemas, la metacognición y la gestión del conocimiento como elementos sustanciales dentro del proceso educativo.

\footnotetext{
2 Tomado de la clase de Metacognición a cargo del profesor Hernán Mera Borrero en el Diplomado de docencia Universitaria de la Institución Universitaria Antonio José Camacho
}

\section{CONCEPTOS PREVIOS INTERCULTURALIDAD}

Este concepto tiene implícito el diálogo y la interacción entre personas y grupos de personas diversos. La Unesco define la interculturalidad como la presencia e interacción equitativa de diversas culturas y la posibilidad de generar expresiones culturales compartidas, a través del diálogo y del respeto mutuo (Unesco, 2020).

El Ministerio de Cultura en Colombia aborda el término interculturalidad en su documento de Políticas de diversidad cultural y referencia cómo, desde el año 1991, el estado colombiano es reconocido como un estado pluriétnico y multicultural; asimismo señala que:

este reconocimiento se ha traducido en normas y políticas y en numerosas acciones institucionales, pero estos esfuerzos son todavía insuficientes para crear una cultura de reconocimiento y respeto por la diferencia, y de diálogo y ejercicio de la interculturalidad en la solución de los problemas que conciernen o afectan a los grupos culturalmente diferenciados. (Ministerio de Cultura de Colombia, 2009)

Este concepto es relevante en la sistematización de la experiencia pedagógica porque, como se verá más adelante, las personas que conforman el grupo tienen diversidad étnica, sexual y cognitiva, y durante el proceso de enseñanza y aprendizaje se generaron diálogos que permitieron la construcción de conocimientos prácticos desde sus historias de vida y su realidad sociocultural.

Raimon Panikkar (2006) afirma que:

El diálogo intercultural se da entre la conversación de las personas y no entre individuos, en cuanto que no se trata solo de un diálogo individual entre dos seres humanos desarraigados de su substrato y de su historia, sino de una ósmosis entre dos visiones de la realidad, más aún entre dos mundos, por así decirlo, por dos personas humanas que llevan consigo todo el peso (la historia) de sus culturas. (Panikkar, 2006) 
Paulo Freyre, por su parte, reconoce el diálogo como una exigencia existencial, pues el encuentro que propicia la reflexión y la acción de sus sujetos no puede reducirse al acto de depositar ideas de un sujeto en el otro. Por el contrario, el diálogo estimula la creación y la transformación intencionada de los sujetos y su entorno (Freire, 1998).

\section{APRENDIZAJE BASADO EN PROBLEMAS (ABP)}

El ABP fue una de las estrategias didácticas implementadas durante el desarrollo del proceso pedagógico sistematizado, cuyo propósito principal fue enseñar a diseñar soluciones creativas a problemas sentidos y reales.

Tanto el estudiante como el profesor asumen unos roles diferenciados pero complementarios que propician una comprensión de la realidad con el fin de transformarla.

Es allí donde se propicia, de manera intencionada, la formación de los estudiantes como líderes y sujetos.

El rol del profesor es de mediador, más no directivo. Diseña o selecciona el problema, la experiencia; acompaña y orienta al grupo en la solución del problema. Por otro lado, el rol del estudiante consiste en ser autónomo y reconocer qué sabe y qué no, qué recursos y fuentes de información se necesitarán para la solución del problema (Samper, 2004).

\section{METACOGNICIÓN}

La metacognición o teoría de la mente es un área de la cognición que aporta a la configuración de nuevas concepciones para los procesos de enseñanza y aprendizaje, de una manera consciente y regulada por parte del sujeto para disponerse a aprender o enseñar (Glaser, 1994).

Flavell (1976: 232), uno de los pioneros en la utilización de este término, afirma que la metacognición, por un lado, se refiere "al conocimiento que un individuo posee acerca de sus propios procesos cognitivos" y, por otro, "a la inspección activa y consecuente regulación y organización de estos procesos" (Flavell, 1976).

La metacognición se aplica tanto para estudiantes como para profesores. En el caso de un estudiante, un ejemplo de metacognición es cuando hay reflexión y conciencia sobre cómo se aprende y, para el caso de los docentes, un ejemplo podría ser cuando hay intencionalidad sobre el uso de las estrategias de enseñanza más adecuadas en cada caso y posteriormente se da una reflexión sobre la práctica, sobre lo que se enseña y se aprende.

La metacognición comprende tres momentos. El primero se relaciona con la estructura del conocimiento y el estado de consciencia que las personas tienen en sí mismas en su papel de aprendices al solucionar problemas con los recursos que cuentan para resolverlo. El segundo momento es el autocontrol y ordenamiento por parte del estudiante de sus propias destrezas cognitivas. El tercer y último momento tiene que ver con la habilidad reflexiva del propio conocimiento adquirido mediante procesos de aprendizaje significativo (Donado, 2007)

La metacognición es por tanto un mecanismo que recrea las condiciones para que resulte un proceso de enseñanza-aprendizaje significativo dado que, dispone la estructura mental del sujeto para que posea ideas de afianzamiento con las que pueda aprender significativamente mediante didácticas contemporáneas como el aprendizaje basado en problemas y proyectos y por otra parte motiva al docente a elaborar material estructurado y lógico relacionado con la estructura de conocimiento (Soro, 2001)

\section{GESTIÓN DEL CONOCIMIENTO}

Las Gestión del Conocimiento GC está fundamentada por todo aquello que permite interpretar los diferentes fenómenos en cada entorno de aprendizaje y, a su vez, está asociado a una serie de estrategias gestadas, bajo la luz de unas didácticas contemporáneas, aisladas de las 
didácticas tradicionales, donde el fenómeno interactivo entre los diferentes actores hace que el aprendizaje trascienda y genere un impacto mayor por los diferentes procesos mediadores que se dan en el aula. Por otra parte, comprende el apoyo de las tecnologías de la información y de las herramientas tecnológicas que cada vez se hacen más potentes en el sector educativo, maximizando la optimización del tiempo, entre la interacción que tiene el estudiante con el docente que lo acompaña (Martínez, 2003).

La GC está indisolublemente ligada con la gestión de las tecnologías. En un sentido estricto es una forma de gestión de la información centrada en un tipo de información, el know-how, que está representada y comunicada de forma exclusiva por las nuevas tecnologías de la información. Si el objetivo de la GC es que el conocimiento sea accesible debe emplear para ello los medios adecuados. Las tecnologías son tan sólo un medio, pero en el caso de la GC parecen tener una relación tan vinculante que no se concibe ésta sin el uso de aquéllas. Las tecnologías de la información son utilizadas en diversos contextos. Una biblioteca virtual, por ejemplo, es conocimiento representado en un entorno virtual.

Según Canals (2003), el conocimiento es "todo lo que nosotros tenemos que nos ayuda a interpretar el entorno y, como consecuencia, a actuar". Teniendo en cuenta esta definición, en los ambientes de aprendizaje se debe contemplar diferentes estrategias didácticas que conllevan a explorar los variados fenómenos que se presentan en el aula, esto permitirá realizar una interpretación del entorno de una manera más crítica sobre lo que se trasforma en cada fenómeno de aprendizaje.

\section{SISTEMATIZACIÓN DE LA EXPERIENCIA}

Para sistematizar la experiencia de la práctica docente se recurrió a un modelo que se muestra en la Figura 1:

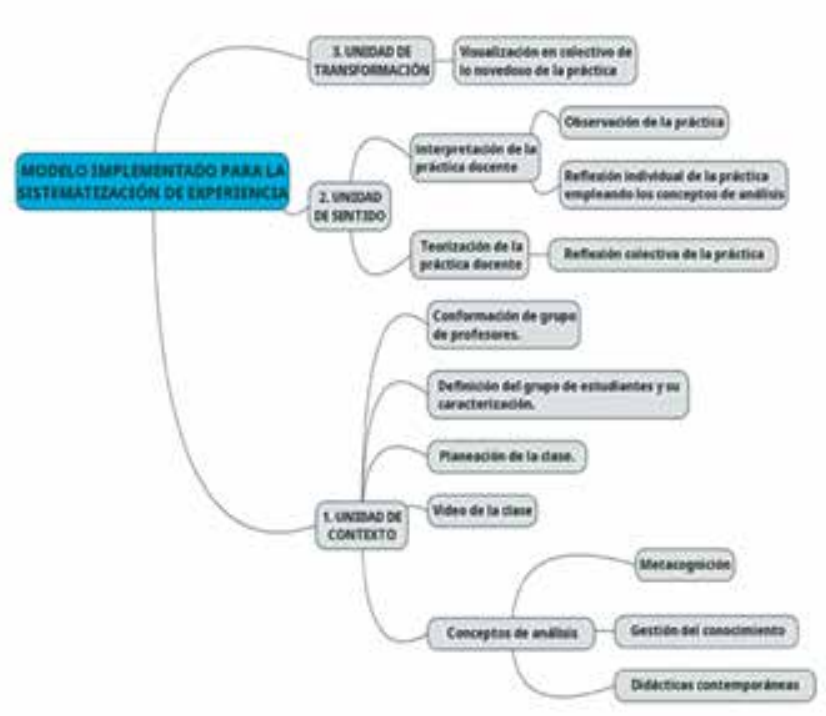

Fig. 1. Modelo propuesto para la sistematización de experiencias. Fuente: Elaboración Propia

\section{UNIDAD DE CONTEXTO}

Dentro de la unidad de contexto, la caracterización del grupo observado, así como la observación apreciativa y crítica de la docente permitieron precisar un aula de clase conformada por un grupo de 26 personas jóvenes, con edades comprendidas entre los 19 y 30 años. Todos inscritos dentro del proceso de formación de la UNIAJC en varios campos disciplinares: Trabajo social, Artes Visuales y Diseño visual, matriculados en la asignatura Iniciativa Empresarial y participando de la unidad de contenido de Innovación Social y Cultural; un grupo conformado en su mayoría por mujeres. Dentro de la caracterización se evidencia diversidad cultural, étnica, de género, y de necesidades educativas especiales. De los estudiantes de trabajo social (10) son afrodescendientes provenientes de Tumaco Nariño, del Charco Nariño, de Timbiquí Cauca y Buenos Aires Cauca, con cosmovisiones diferentes, en proceso de adaptación a la ciudad ya que provienen de zonas rurales. Algunos estudiantes de Diseño Visual son provenientes de Medellín (1) y Cartago (1), los demás son de la ciudad de Cali.

Los estudiantes de Artes y Diseño visual mostraron una mayor apertura a la asignatura, a ser 
emprendedores y empresarios; con los estudiantes de Trabajo social se percibe inicialmente una leve resistencia, lo cual los lleva a preguntarse ¿por qué está en el pensum una asignatura que lleva por nombre Iniciativa Empresarial? Se aclara que es una asignatura que hace parte del proceso de resignificación curricular de la Institución, la cual conlleva a desarrollar procesos de aprendizaje y enseñanza en liderazgo y emprendimiento, asumiendo nuevos paradigmas y modelos educativos desde posturas y concepciones alternativas que reivindiquen el papel de la educación en tiempos de crisis y exclusión. La propuesta de transformar la asignatura y asumir el liderazgo y el emprendimiento desde una nueva perspectiva en la UNIAJC constituye una forma de incidir en la construcción de un mundo más humano y una sociedad con más equidad, y su intencionalidad y su contenido están encaminados hacia el bien común (Castaño, Ortiz, \& Palacio, 2019). Con el desarrollo de la asignatura se enfatizó en una dimensión axiológica y socio afectiva, lo cual propició apertura por parte de los sujetos estudiantes a los encuentros realizados durante el proceso.

La experiencia que se sistematizó tiene como contenido la innovación social y cultural desde 3 dimensiones: individual, desde la problematización personal de su realidad; colectiva y de contexto, a través de las ruedas de intercambio y colaboración que permitieron la concreción de ideas para el cambio y el bien común.

La profesora observada desarrolló los encuentros en el espacio físico de la UNIAJC y para el presente análisis se consideraron las siguientes categorías:

Ambiente físico. El aula de clase estaba dispuesta en forma de herradura, con dispersión en ocasiones debido al mal clima y las llegadas tarde de los estudiantes, sin embargo, se facilitó la rueda de intercambio y colaboración.

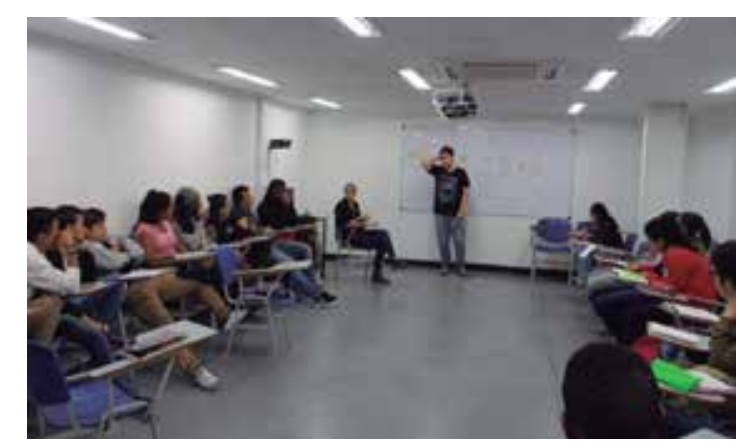

Fotografía 1. Ambiente físico del aula de clase (noviembre de 2018)

Comunicación. La profesora planteó una estrategia comunicativa, con una consigna de trabajo que se repite de manera permanente "escucha activa". Se pondera la participación del grupo de manera individual con aportes y de manera colectiva en el trabajo de subgrupos. Se promueve la participación con la generación de confianza al resolver inquietudes desde preguntas orientadoras. La docente fomenta la sensibilidad intercultural en el aula, entendida ésta como la proyección y respuestas emocionales positivas frente a los demás (Nubía Cecilia Agudelo, 2009), se acerca a los estudiantes, se moviliza por todo el espacio y mantiene una actitud cálida y afectuosa, los escucha y luego les informa. Ofrece instrucciones claras, hace de forma permanente un llamado a la escucha activa, al estar en el aquí y el ahora.

Investigación. Promueve la problematización de la realidad desde las pasiones, motivaciones, dolores y ausencias personales, como una forma de conocer y comprender el contexto y el entorno que se habita desde un sentido crítico y transformador. Se propone la observación, la pregunta como forma exploratoria e indica cómo los problemas formulados tienen una relación directa con los procesos de investigación al convertirse posteriormente en pregunta de investigación o formar parte del proyecto integrador. Se trabajó el intercambio y la colaboración entre los sujetos estudiantes y sus problemas, lo cual permitió un encuentro intercultural y el tejido de unos horizontes posibles. Utilizó la estrategia de búsqueda conceptual 
con apoyo de las TIC con algunos conceptos y categorías que fueron emergiendo durante la clase. Igualmente orienta cómo este trabajo ayuda a reconocer aprendizajes que serán evaluados en el parcial.

Enseñanza. Utiliza estrategias de la secuencia didáctica al recoger el saber anterior, explicar conceptos, aclarar y orientar la intención en la formulación de problemas. Formula de manera permanente las preguntas que orientan el ejercicio y que fomentan una comprensión de la realidad.

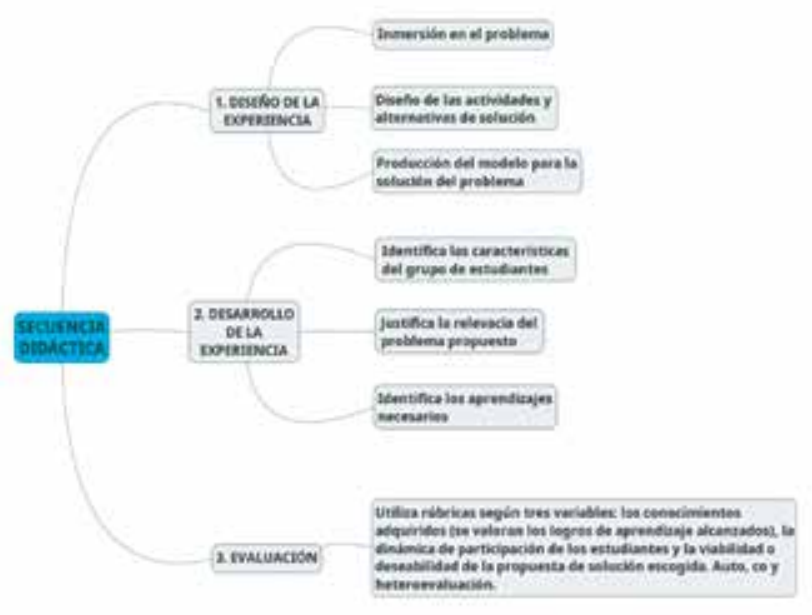

Fig. 2. Modelo propuesto para la secuencia didáctica Fuente: Elaboración Propia

Aprendizaje. Identifica el aprendizaje de los estudiantes, ubica las características socioculturales, invita con preguntas orientadoras y con la instrucción a que los estudiantes indaguen y encuentren el aprendizaje.

Evaluación. Trabaja la evaluación como proceso al retroalimentar la socialización de los problemas e invitar a los estudiantes a participar del proceso evaluativo de los compañeros. Utiliza la estrategia de búsqueda conceptual con el concepto de desarraigo.

Teniendo en cuenta las categorías anteriores, la profesora observada llevó a cabo la siguiente secuencia didáctica para el logro de la intención educativa.
Motivar a los estudiantes por la innovación social y cultural a partir de la identificación de problemas sociales y crear nuevas formas de actuación frente a dicha realidad desde su campo disciplinar.

Secuencia didáctica: Saluda a los estudiantes. Presenta la intención de la clase. Plantea la metodología de trabajo en tres momentos. Desarrolla el trabajo participativo en pequeños subgrupos con la consigna a realizar: identificar un problema sensible a ellos y sus necesidades.

Muestra las condiciones de desarrollo de la clase: Desarrolla la clase en torno a la identificación de problemas sociales para los cuales ellos posteriormente diseñan una solución a través de un producto y/o proyecto sociocultural. Acompaña durante el proceso con preguntas orientadoras o con información pertinente, ofrece finalmente retroalimentación general. Plantea después, el segundo momento, el espacio en donde se va a materializar lo planteado en el problema como un proyecto de innovación social, donde la creatividad e iniciativa sean parte del proyecto. El objetivo fue iniciar el proceso de diseño/ideación de un producto (bien o servicio) cuyo enfoque teórico es la Innovación social y cultural y el modelo de negocio.

En el segundo momento se desarrolló una rueda de intercambio y colaboración para conectar problemáticas, hacer aportes y finalmente se crearon grupos donde se inició el estudio del "modelo de negocio Canvas", en el cual se debía reflexionar para apropiarlo a las iniciativas empresariales de tipo social y cultural. Como estrategias de aprendizaje se emplearon la gestión del conocimiento por medio del uso de las TIC y los pre-saberes, Aprendizaje Basado en Problemas y Metacognición. Como resultados se tiene que cada uno de los estudiantes planteó un problema que aqueja a cierto sector de la sociedad en el ámbito social y/o cultural, el cual, al ser de su interés, los 
impulsa a trabajar en su solución. Con respecto a la planeación inicial, no se lograron cumplir los tiempos programados para cada actividad ni abarcar todos los contenidos. Como dificultades se encontró que las condiciones climáticas retrasaron el inicio de las clases, los estudiantes llegaban tarde y demoraban en conectarse con la clase.

Dentro de la unidad de contexto, se indica la reflexión sobre los septenios de la profesora observada, se parte de la idea: "cuanto más trabajamos en nuestra propia biografía y más la comprendemos, mejor entendemos a las demás personas", tomada del libro El hilo de la vida de Gundrun Burkhard.

Es importante resaltar como el trabajo de introspección del docente influye en el proceso de enseñanza y aprendizaje en el aula de clase. Los septenios van desde el primer al cuarto septenio, pero en el presente artículo solo se presentan los dos primeros.

La primera obra es una representación biográfica con resignificación incluida, denominada Entre miedos y caricias (Anónimo, 2018), donde aparecen dos representaciones a manera de contraste. En la primera:

Se teje la individualidad alrededor del cuerpo físico y de la forma como los otros lo perciben y lo construyen con ella. Crece en un hogar rodeado de valores como la honestidad, el compromiso, el orden y la limpieza. Las actividades que más disfruta son al aire libre en compañía de su padre, con quien se hace más fuerte el vínculo afectivo, Durante este septenio hay un sentimiento de nostalgia, de timidez, de inseguridad y miedo. (Anónimo, 2018)

La segunda obra es "Y Dios me hizo mujer" (Anónimo, 2018), representa la posibilidad de darle un nuevo significado a los acontecimientos positivos y negativos vividos durante este septenio es una obra inspirada en las experiencias espirituales, el relacionamiento con el yo, las relaciones y vínculos con el entorno y la fuerza emocional que brindan los años. (Anónimo, 2018)

Cada colección contiene, además, 2 fotografías de la Autora, una al comienzo y otra al final del septenio.

Es importante señalar cómo en el primer momento de la unidad de contexto, tanto la caracterización del grupo, como la secuencia didáctica que la docente desarrolló tuvieron una estrecha relación, es decir, que la docente conocía la realidad que habita su grupo y desde allí dinamizó las diferentes estrategias didácticas que propiciaron la reflexión y análisis. De ahí que la intención pedagógica del aprendizaje basado en problemas tejido a las realidades del entorno circundante de los participantes del grupo, ponen en el terreno del aprendizaje significativo la necesidad de explorar las realidades sociales y ofrecer innovación cultural social, como caminos a seguir dentro de las iniciativas empresariales, el liderazgo y la gestión de procesos de cambio.

Es cierto que dentro de la apuesta de las didácticas contemporáneas el aprendizaje basado en problemas sitúa la relación de profesor-estudiante en el terreno de la participación, donde se recoge el saber acumulado de la experiencia y se articula a los nuevos saberes como una forma de inclusión y gestión del conocimiento. En el mismo sentido, el trabajar bajo la intención de la enseñanza basada en problemas pone de manifiesto, sin darnos cuenta, cómo los problemas de la realidad social y cultural que se plantearon en el aula de clase, mostraron el entorno real que circunda a los participantes donde el componente socio-afectivo apareció en la medida en que los problemas se manifestaron como sentires particulares y propios de la comunidad de estudiantes, tales como los problemas de género, exclusión social, necesidades de reconocimiento y cómo este sentir fue transmitido al cuerpo a través de los canales de comunicación oral, escrito y gráfico, y analizado en el cerebro en el espacio 
de la mente, donde los estudiantes logran caracterizar el problema, identificar las unidades de este y construir líneas de acción en torno a salidas y maneras de afrontarlo desde el liderazgo y la innovación social y cultural.

El seguir esta secuencia de análisis a partir de las instancias del entorno, cuerpo, cerebro y mente y precisar el proceso como parte del desarrollo necesario en el aula para que los procesos de metacognición se den de forma permanente. Sin embargo, se hace necesario establecer una mayor conciencia de que este proceso se está fomentando en el aula y está propiciando el aprender a aprender.

De igual forma, la reflexión sobre lo observado precisó como en la experiencia didáctica se crearon las condiciones para el fomento de los potenciales del desarrollo humano, ya que a partir de la elaboración de problemas y sus posibles acciones de solución, los componentes socio afectivo, cognitivo comunicativo, ético, político, entre otros, ganaron sentido en la medida que la búsqueda, la discusión, el desarrollo de una postura y el discurso ético y político se situaron en el desarrollo del encuentro.

\section{UNIDAD DE SENTIDO}

Dentro del proceso de análisis de la experiencia didáctica aparece de manera seguida a la unidad de contexto la unidad de sentido, la cual hace referencia a la relación que se establece en dos procesos significativos: la interpretación de la práctica en el aula donde gana sentido el proceso de observación individual y la correspondiente reflexión de cada uno de los profesores observadores, en donde la reflexión colectiva gana sentido en la medida en que orienta la discusión y la reflexión posterior, ofreciendo la oportunidad de la teorización de la práctica, en donde los hallazgos se relacionan y asocian con conceptos válidos de la teoría dentro del proceso de sistematización.

En este segundo momento de la unidad de sentido, se recogen algunos elementos de interpretación de la experiencia didáctica a manera de datos y hallazgos que al asociarse con conceptos de la práctica pedagógica permiten ilustrar la teoría que surge de dicha reflexión. Todo lo cual se ilustra en la siguiente tabla.

\begin{tabular}{|c|c|}
\hline INTERPAETACOÓN DE LA PRÁCTCA & TECRIZACINN DE LA PELLCTICA \\
\hline 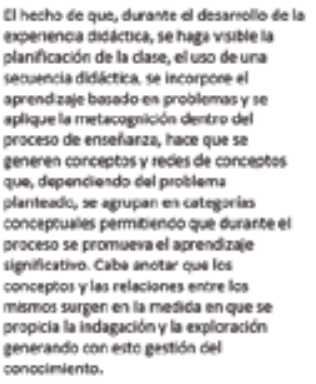 & 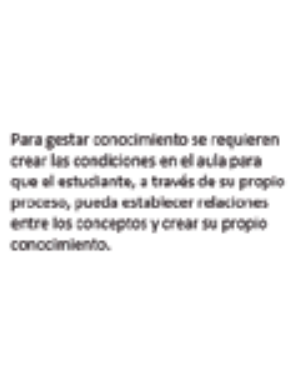 \\
\hline 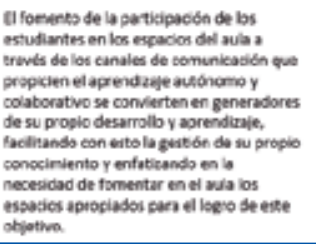 & 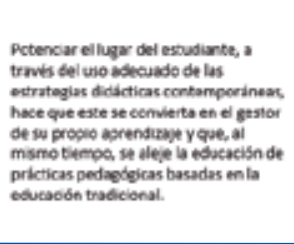 \\
\hline 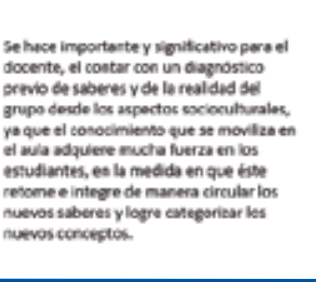 & 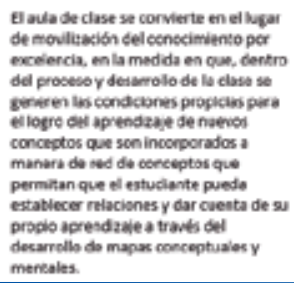 \\
\hline 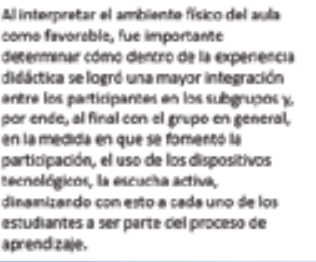 & 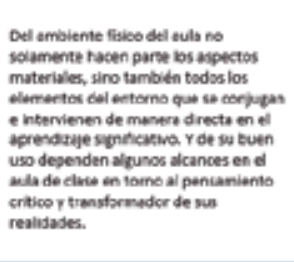 \\
\hline 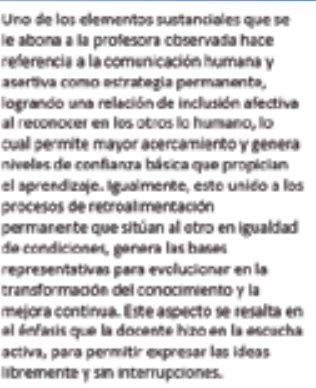 & 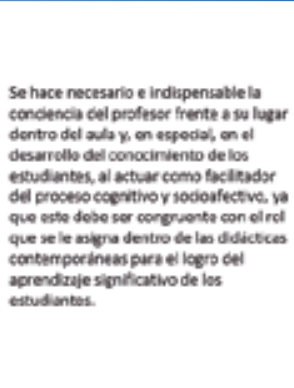 \\
\hline
\end{tabular}

Tabla 1. Unidad de sentido, interpretación de hallazgos. Posible teorización

Fuente: Elaboración Propia

La relación entre lo interpretado en el aula de clase por cada uno de los profesores observadores, durante el desarrollo de la experiencia didáctica y la posible teorización que se logra visualizar, indica cómo esta unidad gana sentido en la medida en que la 
interpretación por sí sola no ofrece lo esencial y sustancial de la experiencia, sino que, por el contrario, la reflexión, el análisis crítico, la mirada conceptual, permite que se alcance a definir elementos teóricos que por sí solos van más allá de la misma experiencia. La interpretación de los hallazgos en el aula de clase, unido al pensar sobre lo que se hace posibilitando la teorización, muestra el camino a seguir dentro de toda práctica docente, en donde se resalta el lugar del profesor como facilitador de los procesos y promotor de la gestión del conocimiento como parte fundamental del aprendizaje significativo.

\section{UNIDAD DE TRANSFORMACIÓN}

Esta unidad indica dentro de la reflexión colectiva lo novedoso y significativo de la experiencia pedagógica, guiando el proceso de sistematización de la práctica docente e indicando los pasos a seguir dentro del análisis que conlleva a una mirada retrospectiva y transformadora del lugar del profesor en el aula y del significado de su papel como agente transformador y generador de conocimiento. Además, hace referencia a:

- Atravesar la frontera social (conexión emocional)

- $\quad$ Aura (Aquí y ahora).

- Rueda de intercambio y colaboración.

- $\quad$ Promoción escucha activa.

- $\quad$ Caracterización del grupo.

- $\quad$ Emergen redes de conceptos a partir del tópico generativo.

- No hay una relación de poder.

En ese sentido, esta unidad invita a pensar en la práctica docente como una experiencia de vida que traspasa los linderos de la educación tradicional, la transmisión del conocimiento, los escenarios de poder y control del saber y el aprendizaje y crea las condiciones para que se pondere el lugar del que aprende y se fomente el aprender a aprender. Para lo cual nos permitimos definir:

- Toda experiencia en el aula debe estar acompañada de una clara estrategia didáctica.

- Todo proceso de aprendizaje debe potenciar la gestión del conocimiento y motivar el aprender a aprender.

Los procesos metacognitivos son esenciales en el proceso de gestión del conocimiento.

- El diálogo de saberes en el aula desde las prácticas y miradas de cada estudiante, desde su diversidad, desde su singularidad en un ambiente de confianza, de entusiasmo y de interés por inzfluir en el mundo que habita es fundamental en la construcción de subjetividad.

- El proceso vivenciado en la asignatura Iniciativa Empresarial estuvo orientado a la formación de líderes sujetos políticos.

- Dentro de todo proceso de aprendizaje el entorno, el cuerpo, el cerebro y la mente mantienen una conexión permanente y una circularidad constante que requiere ser tenida en cuenta en cada experiencia didáctica.

- La sistematización de la experiencia didáctica y su modelo es fundamentalmente útil y esencial dentro de la evaluación de la práctica docente.

Es evidente como en la clase la docente da mayor importancia al sujeto que aprende que al objeto de aprendizaje.

\section{CONCLUSIONES}

Sistematizar fenómenos presentados en las aulas de clase permite tener un análisis crítico y reflexivo 
sobre el comportamiento de estudiantes y profesores en los escenarios educativos, esto posibilita ampliar horizontes sobre las formas $y$ métodos de aprender y enseñar, trascendiendo de las metodologías tradicionales a las contemporáneas y permitiendo explorar de una manera significativa el componente humano entre las partes involucradas en la transformación del conocimiento. Ahora bien, sistematizar escenarios académicos y reflexionar sobre estos contribuye a que el desarrollo profesoral sea más eficiente y efectivo, pues este nos permite realizar una observación directa e interdisciplinar entre profesionales con competencias y habilidades potencialmente desarrolladas, aportando a la mejora continua de los docentes observados y retro-alimentando a los observadores para no caer en una mala práctica en su ejercicio docente o profesoral.

La sistematización de una experiencia en el aula genera un mayor interés por revelar aspectos invisibilizados de la práctica, tales como las conexiones emocionales, las relaciones de poder y un cuestionamiento crítico de las propuestas pedagógicas $\mathrm{y}$ didácticas que se han venido utilizando.

Dentro de la práctica educativa se hace necesario implementar el modelo de sistematización de la experiencia didáctica en el aula como una forma de auto-evaluación y coevaluación, donde se reflexiona, analiza y teoriza de manera individual y colectiva el quehacer en el aula, en bien del aprendizaje significativo y la gestión del conocimiento como elementos sustanciales dentro de los procesos de formación docente.

Para educar estudiantes metacognitivos se requiere de docentes metacognitivos. Esto se logra cuando los profesores adecuan sus prácticas pedagógicas en el aula, siendo conscientes de sus potenciales de desarrollo humano y sus limitaciones.
Como resultado del proceso de sistematización de experiencia, se obtiene una reflexión sobre la labor de educar, que permite a los profesores llegar a regular sus estrategias de enseñanza, y puedan acercarse a la meta de "enseñar a aprender" a sus estudiantes, orientando el proceso educativo hacia un aprendizaje significativo y autónomo que les conduzca a "aprender a aprender".

\section{REFERENCIAS BIBLIOGRÁFICAS".}

Anónimo. (2018). Entre miedos y caricias. Obra inédita. Anónimo. (2018). Y Dios me hizo mujer. Obra inédita.

Agudelo Cely, N. C. y Estupiñán Quiñones, N. (2009). La Sensibilidad Intercultural en Paulo Freire. Revista Historia de la Educación Latinoamericana, (13), 85-100.

Canals, A. (2003). La gestión del conocimiento Universitat Oberta de Catalunya. http://www. uoc.edu/dt/20251/index.html

Castaño, M. M., Ortiz, H., y Palacio, Z. (2019). Liderazgo y Emprendimiento en la Uniajc hacia una nueva perspectiva. Cali: Institución Universitaria Antonio José Camacho.

Donado, L. G. (2007). Procesos metacognitivos de estudiantes con diferentes estilos de aprendizaje (tesis de maestría). Universidad del Norte, Barranquilla, Colombia.

Flavell, J. (1976). Metacognitive aspects of problem solving. En: L. B. Resnik (ed.). The nature of intelligence (pp. 231-235). Hillsdale, N.J.: Erlbaum.

Freire, P. (1998). Pedagogía del oprimido. México: Siglo XXI Editores.

Glaser, R. (1994). Learning theory and instruction. En: G. D'Ydewalle, P. Eelen y B. Bertelson (eds.). International perspectives on psychological science (Vol. 2) NJ: Erlbaum. 
Martínez, F. J., Peñalver A. y Salamanca J. (2003). Gestión estratégica del conocimiento. Obtenido de https://auip.org/images/stories/DATOS/Pub licacionesOnLine/archivos/gestionconocimiento .pdf

Mejía, M. R. (2007). La sistematización como proceso investigativo o la búsqueda de la epistemede las prácticas. Revista Internacional Magisterio (33). Obtenido de http://www. cepalforja.org/sistem/sistem_old/sistematizacio n_como_proceso_investigativo.pdf

Ministerio de Cultura de Colombia. (2009). Política de Diversidad Cultural. Obtenido de https://www.mincultura.gov.co/ministerio/pol iticas-culturales/de-diversidad-cultural/Docu ments/07_politica_diversidad_cultural.pdf

Panikkar, R. (2006). Paz e interculturalidad, una reflexión filosófica. España: Herder Editorial.

Samper, M. D. (2004). Enfoques pedagógicos y didácticas contemporaneas. Bogotá: Fundación Internacional de Pedagogía Conceptual.

Soro, M. B. (2001). Estrategias metacognitivas y de aprendizaje: estudio empírico sobre el efecto de la aplicación de un programa metacognitivo, y el dominio de las estrategias de aprendizaje en estudiantes de E.S.O, B.U.P y universidad (tesis de doctoriado). Universidad Complutense de Madrid, Madrid, España.

\section{AUTORES}

Mónica María Castaño Castaño: profesional en Gestión Cultural y Comunicativa, Universidad Nacional de Colombia. MSc (C) en Derechos Humanos y Cultura de paz, Pontificia Universidad Javeriana de Cali, Colombia. Docente Ocasional Tiempo Completo, Institución Universitaria Antonio José Camacho, Colombia. Directora del Programa de Liderazgo y Emprendimiento, Institución Universitaria Antonio José Camacho, Colombia. Correo: mcastano@admon.uniajc.edu.co

Lady Jhoanna García García: ingeniera Física, Universidad Tecnológica de Pereira, Colombia. MSc. en Instrumentación Física, Universidad Tecnológica de Pereira, Colombia. Docente Hora Catedra, Universidad Tecnológica de Pereira, Colombia. Correo: lady.garcia@utp.edu.co

Alberto José Echeverría Reina: ingeniero Mecatrónico, Universidad Autónoma de Occidente, Colombia. Especialista en Automatización de Procesos Industriales, Universidad de los Andes, Colombia. Msc. Energías Renovables y Eficiencia Energética, Universidad a Distancia de Madrid, España. Docente Tiempo Completo, Institución Universitaria Antonio José Camacho, Colombia. Correo: aecheverria@admon.uniajc.edu.co

Ana María Becerra Ocampo: licenciada en Artes Visuales de la Universidad del Valle, Colombia. Diplomado en Formación para la primera infancia Universidad San Buenaventura, Colombia. Correo: anamariabecerraocampo@gmail.com

Henry Alonso Ocampo Marín: administrador en Salud, Institución Universitaria Antonio José Camacho, Colombia. MBA (C) Administración y dirección de empresas, Escuela de Negocios Europea de Barcelona ENEB en convenio con la Universidad Isabel I, España. Docente Hora Cátedra, Institución Universitaria Antonio José Camacho, Colombia. Correo: henry.ocampo@fvl.org 